\title{
Field Quality of the Short Superconducting Dipole Models for the LHC
}

\author{
W. Venturini Delsolaro, Z. Ang, L. Bottura, S. Sanfilippo, A. Siemko, D. Tommasini, and L. Walckiers
}

\begin{abstract}
A full characterization of the magnetic field in warm and cold conditions was performed as a part of the standard test on the LHC 1-m long superconducting dipole models. Furthermore, dedicated measurement campaigns addressed the effect of current cycles and quenches on field quality. Powering and quenches were found to generate characteristic instabilities in the geometric harmonics. Detailed results are presented on this phenomenon, as well as correlations between warm and cold measurements and field reconstructions.
\end{abstract}

Index Terms-Field quality, superconducting magnets.

\section{INTRODUCTION}

$\mathbf{T}$ HE LHC short dipole program was mainly dedicated to study the power performance of some variants of the LHC dipole design [1]. Prestress targets determined the choice of pole shims and thus the coil sizes. On the other hand, the performance of the LHC requires accuracy and stability of the field harmonics of the main dipoles to a level where coils deformations due to temperature, prestress and Lorenz forces contribute. The magnetic field quality of model magnets was systematically measured at room and cryogenic temperatures. We report here measurement results on the last eight twin aperture short dipole models with six-block design, called MBSMT magnets. The collaring parameters of MBSMT magnets are summarized in Table I. The other characteristics of these magnets are reported in [1].

\section{EXPERIMENTAL}

Magnetic measurements were performed in vertical cryostats at temperatures ranging from $1.7 \mathrm{~K}$ to $1.9 \mathrm{~K}$. Details on the magnetic measurement system are given in [2]. In short models the end effects were found to influence strongly the field harmonics integrals. Therefore special measuring shafts, featuring central coils $116 \mathrm{~mm}$ long, were built, allowing to isolate the contribution of the straight part of the magnet to the field quality. $116 \mathrm{~mm}$ is the twist pitch of the LHC Rutherford cable. The measurements reported in this paper were taken with the central coil. The magnetic field at cold was measured at different currents between $100 \mathrm{~A}$ and the quench level ("full load line"). Standard measurements were always carried out at the end of the magnet training, as the geometric field quality is unstable during

Manuscript received September 25, 2001.

W. V. Delsolaro, L. Bottura, S. Sanfilippo, A. Siemko, D. Tommasini, and L. Walckiers are with CERN, CH-1211 Geneva 23, Switzerland.

Z. Ang is with TRIUMPH, Vancouver, BC, Canada.

Publisher Item Identifier S 1051-8223(02)03479-6.
TABLE I

COLLARING FEATURES OF MBSMT MAGNETS

MAGNET Pre-stress after collaring [MPa] Pole Shims $[\mathrm{mm}]$ Inner Layer Outer Layer Inner Layer Outer Layer

$\begin{array}{lllll}\text { MBSMT4.V1 } & 56 & 86 & 0.15 & 0.25 \\ \text { MBSMT5.V1 } & 56 & 69 & 0.05 & 0.23 \\ \text { MBSMT6.V1 } & 50 & 93 & 0.05 & 0.23 \\ \text { MBSMT7.V1 } & 59 & 87 & 0.1 & 0.18 \\ \text { MBSMT8.V1 } & 62 & 89 & 0.0 & 0.08 \\ \text { MBSMT9.V1 } & 70 & 83 & 0.0 & 0.08 \\ \text { MBSMT10.V1 } & 54 & 94 & 0.0 & -0.03 \\ \text { MBSMT11.V1 } & 64 & 77 & -0.15 & -0.23\end{array}$

the first training quenches (see Section IV). A standardized current cycle up to $11750 \mathrm{~A}$, corresponding to nominal dipole field of $8.33 \mathrm{~T}$, was performed before each load line measurement, to bring the superconducting filaments into a well reproducible state of magnetization. The magnet current was then ramped up, stopping at pre-defined plateaus (load line currents), where the field was measured after 1 minute waiting time to allow decay of the eddy currents generated during the ramp. A direct current-current transducer (DCCT) provided precise reading of the magnet current. Warm measurements have been carried out at $300 \mathrm{~K}$ with the same test set up, supplying the magnet with \pm 30 A to cancel the residual iron magnetization. As customary, we express the two-dimensional magnetic field in the straight part of an accelerator magnet as a complex Taylor series whose coefficients are the field multipoles expressed in units at the reference radius [3]. Following [4], we considered three different contributions to the field multipoles, i.e., cable positioning errors in the magnet cross-section, magnetization of the superconducting filaments, and saturation of the iron yoke.

Geometric harmonics are defined as averages between the two branches of the hysteresis cycles at $5 \mathrm{kA}$. Magnetization and iron saturation effects are quantified by the deviations from this average at the injection current $(760 \mathrm{~A})$ and at the nominal current respectively. The three contributions are illustrated in Fig. 1 for the normal sextupole.

\section{STANDARD FIELD QUALITY Results}

\section{A. Measurements}

The allowed harmonics averaged over the family of magnets considered are summarized in Table II. Noteworthy is the high value of the average geometric b3, which is comparable with the one found in the first 15-m-long prototypes [5]. A geometric b2 is typical for twin magnets, appearing with opposite sign 


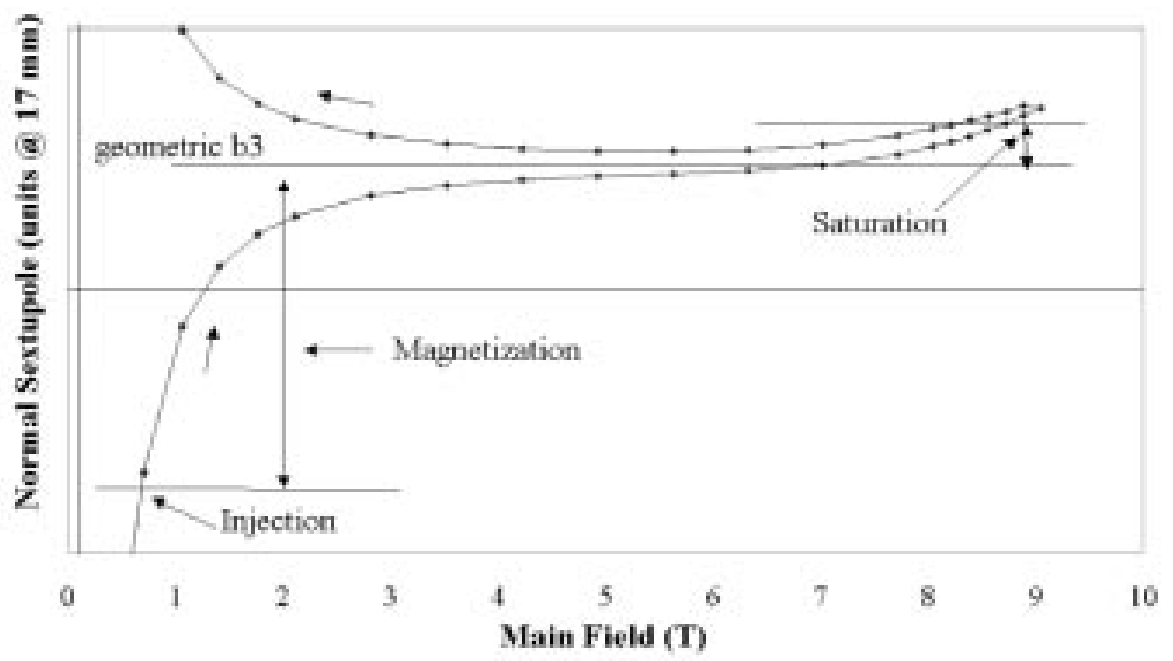

Fig. 1. Hysteresis loop of the normal sextupole in the MBSMT10.V3 magnet, with the three contributions to the field multipole.
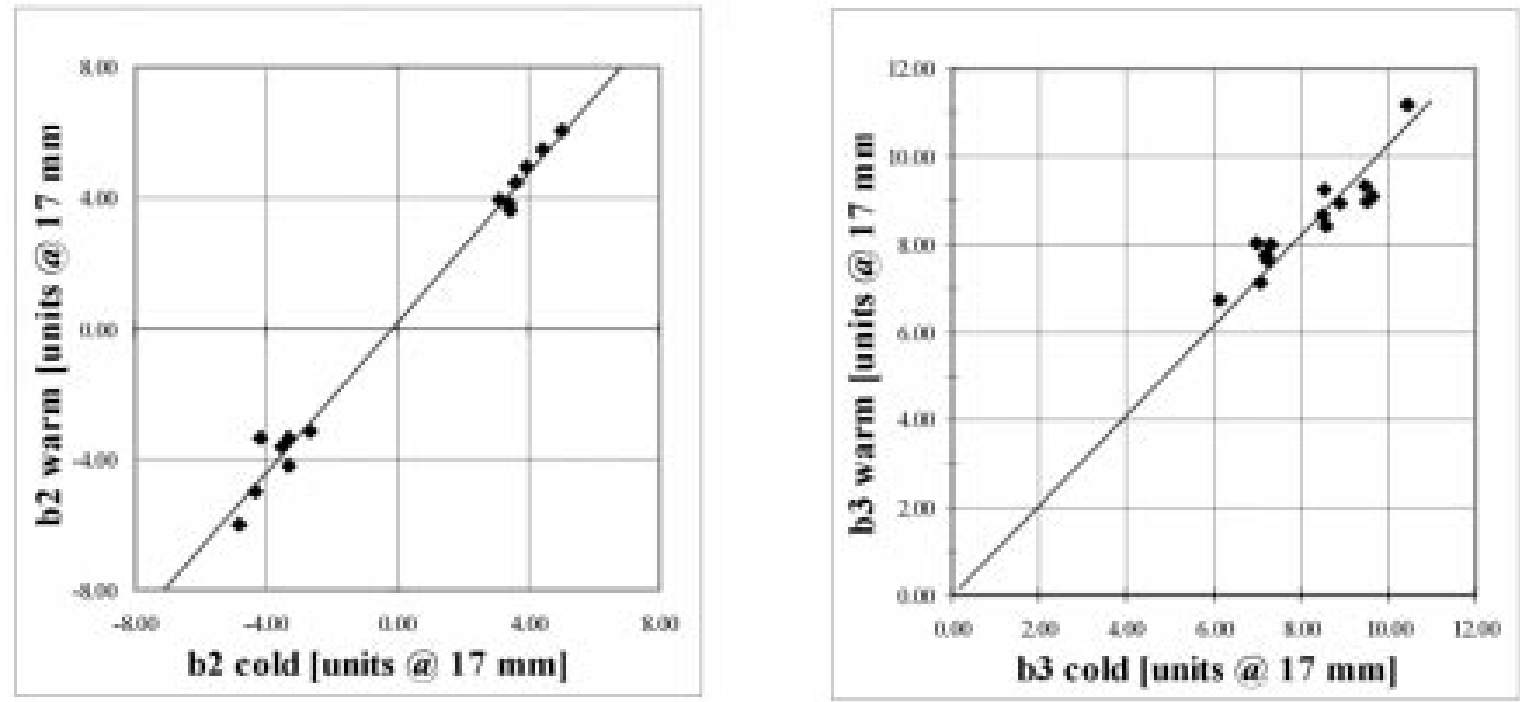

Fig. 2. b2 and b3 warm-cold correlations.

TABLE II

Average Field Multipoles of MBSMT Magnets

\begin{tabular}{ccccccc}
\hline Harmonics & $|b 2|$ & $\mathbf{b 3}$ & $\mathbf{b 5}$ & $\mathbf{b 7}$ & $\mathbf{b 9}$ & $\mathbf{b 1 1}$ \\
\hline Ave. Geom. & 3.62 & 8.26 & 0.41 & 0.73 & 0.19 & 0.58 \\
$\sigma($ geom) & 0.96 & 1.60 & 0.64 & 0.17 & 0.13 & 0.14 \\
Ave. magn. & 0.09 & -7.67 & 1.11 & -0.3 & 0.20 & 0.01 \\
$\sigma($ magn.) & 0.24 & 0.85 & 0.29 & 0.24 & 0.04 & 0.03 \\
Ave. sat & 4.85 & -0.26 & -0.21 & 0.00 & -0.05 & 0.01 \\
$\sigma$ (sat.) & 2.27 & 0.69 & 0.09 & 0.05 & 0.02 & 0.01 \\
\hline
\end{tabular}

in the two apertures (see Fig. 2). The table reports the average absolute values of $\mathrm{b} 2$. The high scatter of data is mainly due to the variety of coil sizes issued from the implementation of the different design variants. The warm cold correlations are shown in Figs. 2 and 3.

\section{B. Analysis}

As for the previous generations of dipole models [6], the geometric multipoles were reconstructed starting from the collaring data of shimming and prestress. The deformation of the collars was estimated according the following equation, valid for stainless steel collars [7]

$$
\delta_{\text {coll }} \cdot[\mathrm{mm}]=0.0036 \sigma
$$

where $\sigma$ is the prestress in MPa averaged between the two layers. The variation of azimuthal coil size was then calculated, as in [6], by $\mathbf{a}-\mathbf{a}_{0}=1 / 2 \delta_{\text {coll }}$. $-\delta_{\text {shim }}$, where $\mathbf{a}$ and $\mathbf{a}_{0}$ are the real and the nominal azimuthal coil sizes, respectively, $\delta_{\text {coll }}$. is the collar expansion, and $\delta_{\text {shim }}$ is the shim thickness variation with respect to the nominal value. The sensitivity (jacobian) of the first allowed harmonics to azimuthal coil size variations was calculated by means of a simulation program [8]. Table III reports the calculated slopes of geometric harmonic dependences 


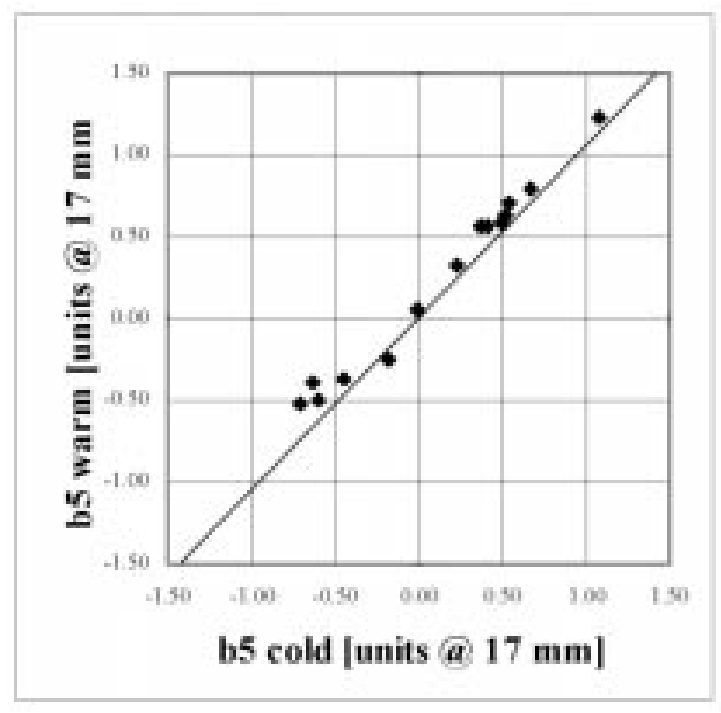

Fig. 3. b5 and b7 warm-cold correlation.

TABLE III

Calculated Slopes of Geometric Harmonics Dependences on AZIMUTHALCOIL SIZE VARIATIONS IN THE INNER AND IN THE OUTER LAYER

\begin{tabular}{ccccc}
\hline $\begin{array}{c}\text { Slopes (units/ } \\
0.1 \mathrm{~mm})\end{array}$ & $\delta \mathrm{b} 3$ & $\delta \mathrm{b} 5$ & $\delta \mathrm{b} 7$ & $\delta \mathrm{b} 9$ \\
\hline Inner layer & -1.716 & 0.362 & -0.1276 & 0.0476 \\
Outer layer & -1.252 & 0.0724 & 0.01408 & -0.00207 \\
\hline
\end{tabular}

on azimuthal coil size variations. These results were used to recalculate the geometric harmonics, only considering the deformation mode of azimuthal stretch in the inner and in the outer layers. The twin aperture magnets behave in this respect as their single aperture counterparts, displaying comparable dispersions around the ideal correlation line. As an example, the case of the normal sextupole is shown in Fig. 4. A similar result is obtained for b7, whereas for b5, the correlation is less good, as shown in Fig. 5.

\section{STABILITY STUDIES}

\section{A. Motivation}

One of the issues so far unresolved in relation with the field quality of the LHC superconducting magnets is the degree of geometric stability to be expected throughout the life of the accelerator. The coils are subjected to large electromagnetic forces that can lead to settling of the cable stacks in the collars. The forces are gradually applied and released during magnet charging and discharging, and they are abruptly released in case of quench. Such perturbations have an effect on the geometric field quality.

\section{B. Experiment and Results}

1) Training of the Field Quality: In order to follow the evolution of the magnet field quality in the early stages of training, magnetic measurements were carried out before and after the first quench, after the second, and after the third quench. The
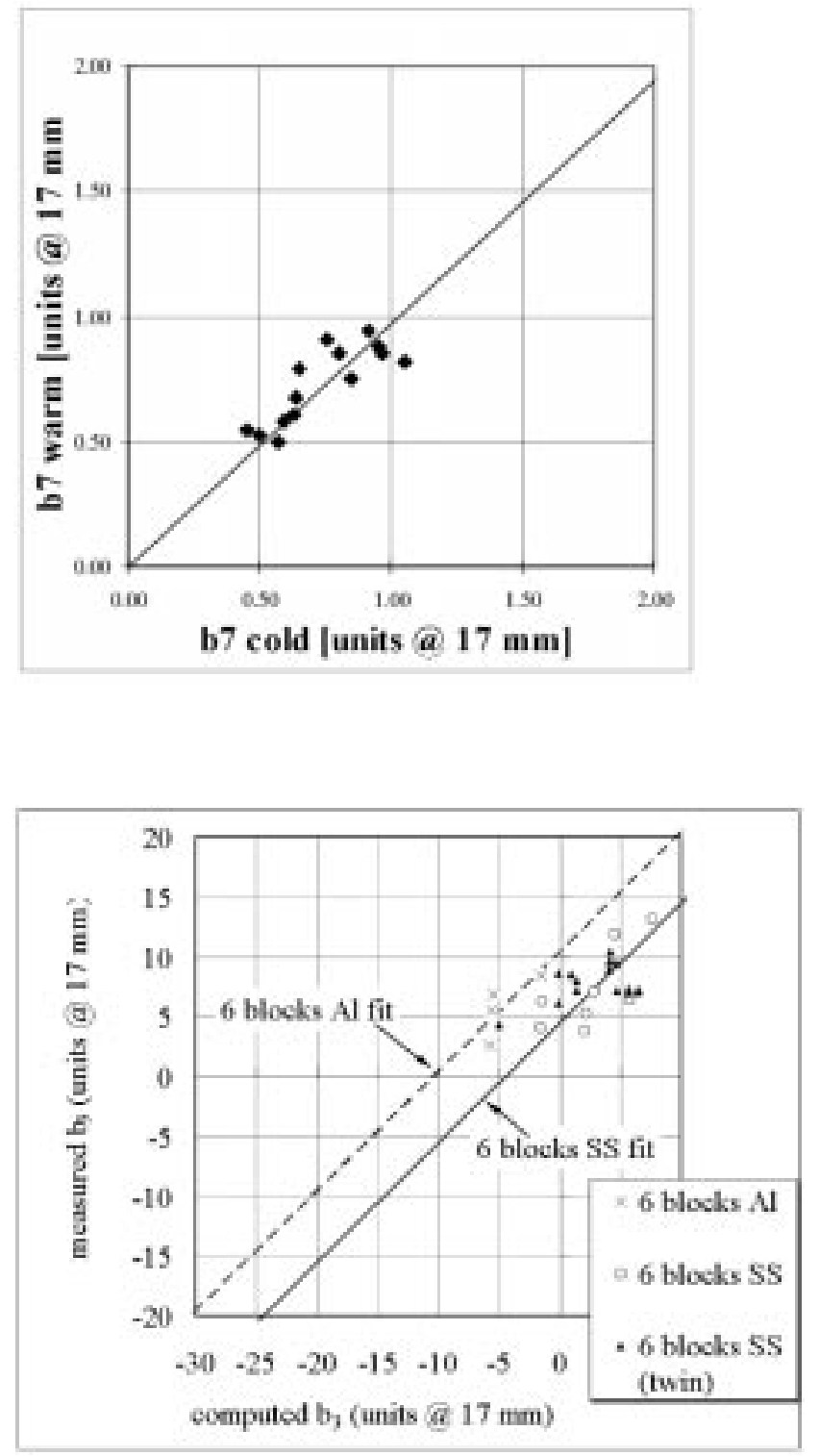

Fig. 4. Field reconstruction: b3 calculated versus b3 measured for three families of short model dipoles.

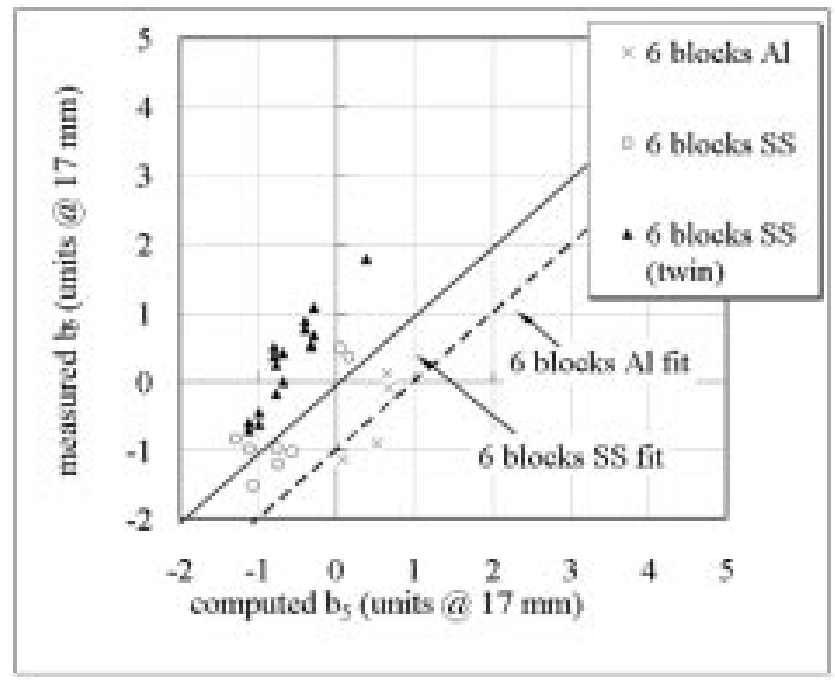

Fig. 5. Field reconstruction: b5 calculated versus b5 measured. 


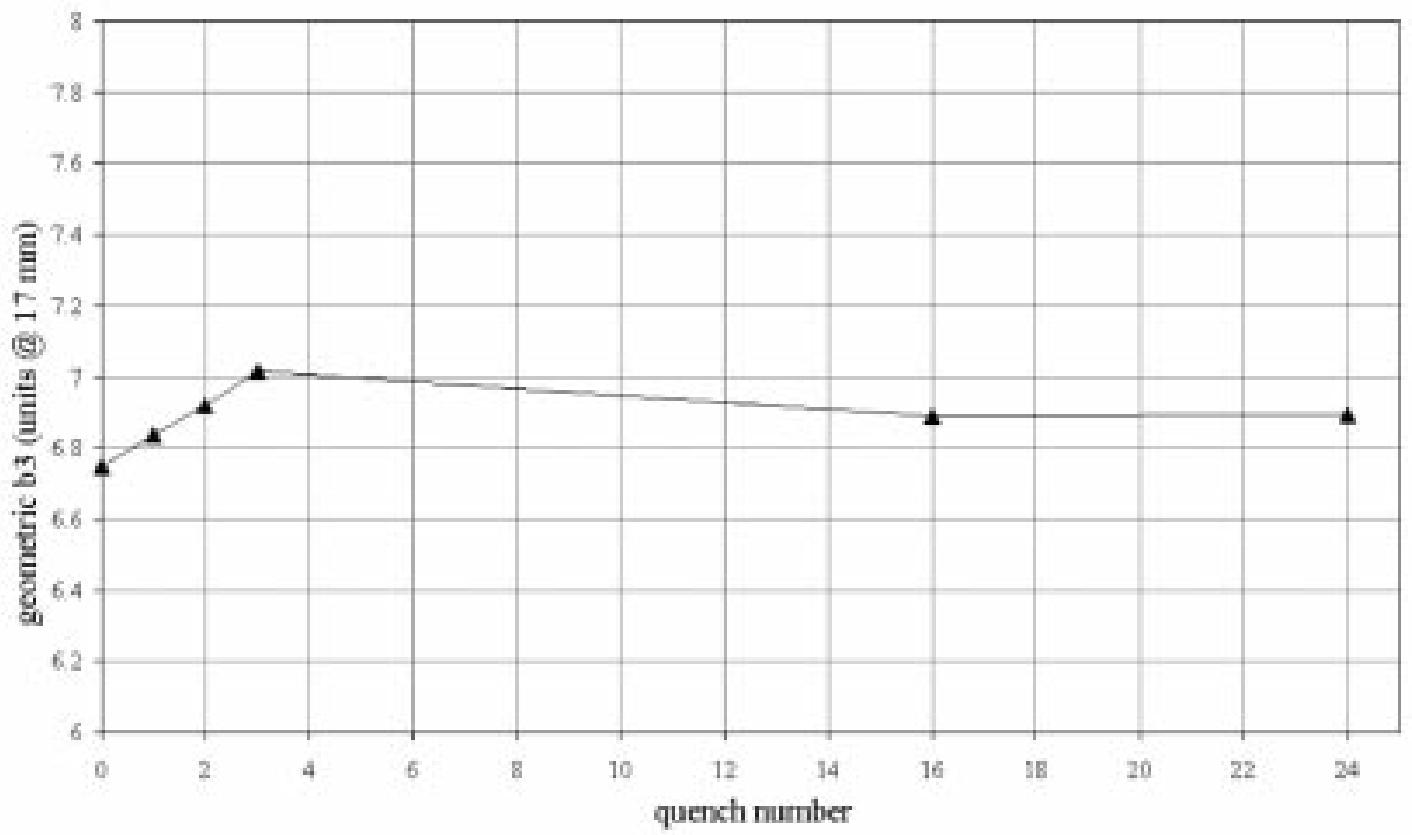

Fig. 6. Evolution of the geometric normal sextupole during training.

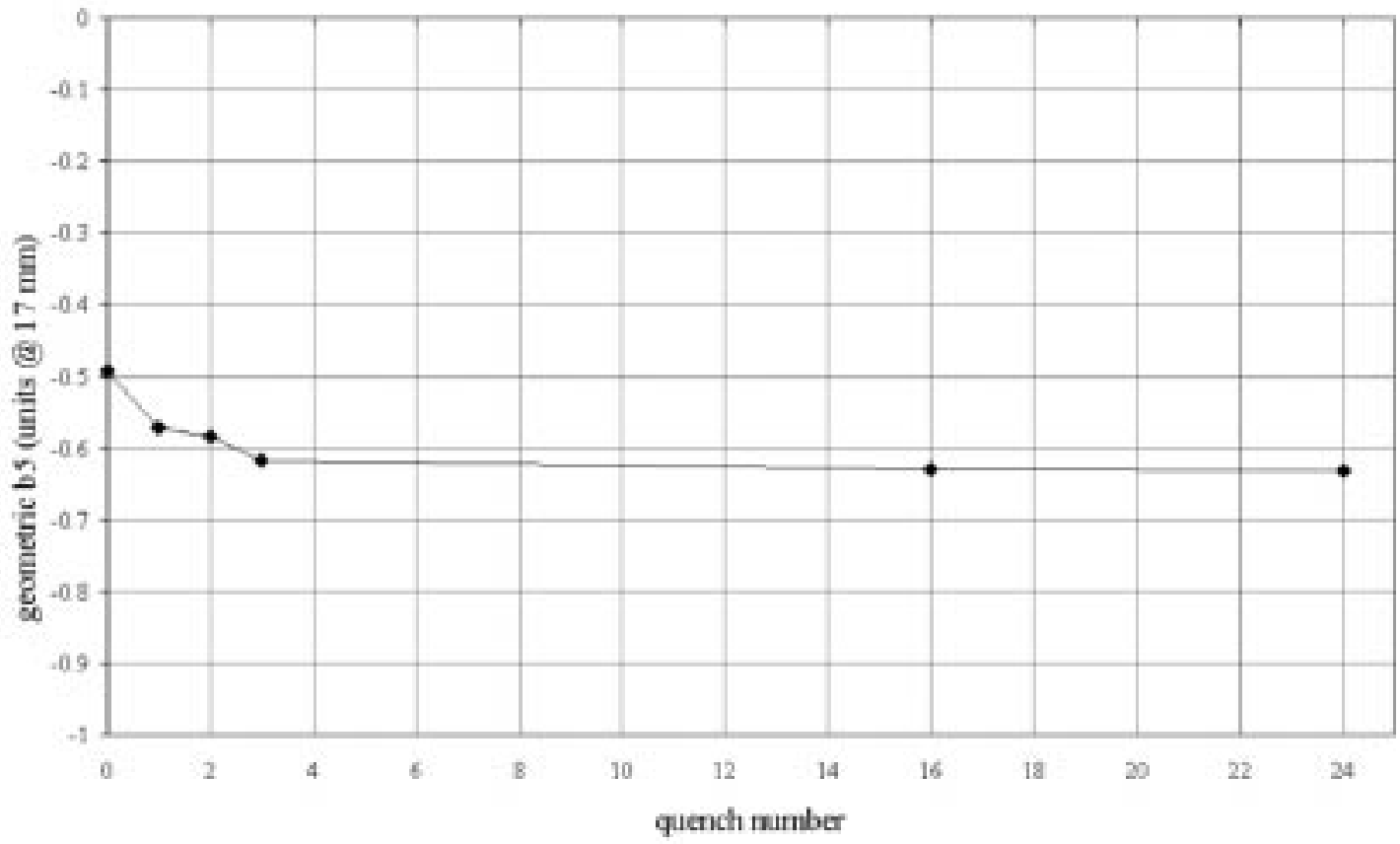

Fig. 7. Evolution of the geometric normal decapole during training.

field was then measured again at the end of the training. This test was executed on a subset of the family of magnets considered here (see Table IV).

A clear trend showed up for the allowed harmonics. In Figs. 6 and 7, the geometric sextupole and decapole are shown as a function of number of training quenches for the MBSMT4.V1. Similar results were obtained for the other magnets: in Table IV we summarize the geometric harmonics variations due to training. The values are maximum excursions defined by $\Delta \mathrm{bi}=\max (\mathrm{bi})-\min (\mathrm{bi})$ during training.

The last digit in the magnet names refers to the aperture.

2) Accelerated Life Test: The field quality of a few magnets was measured as a function of the powering history, to study the effect of cyclic mechanical loads on the geometric harmonics.

In a typical test the measurement of the magnetic field was alternated with series of current cycles between $760 \mathrm{~A}$ and 


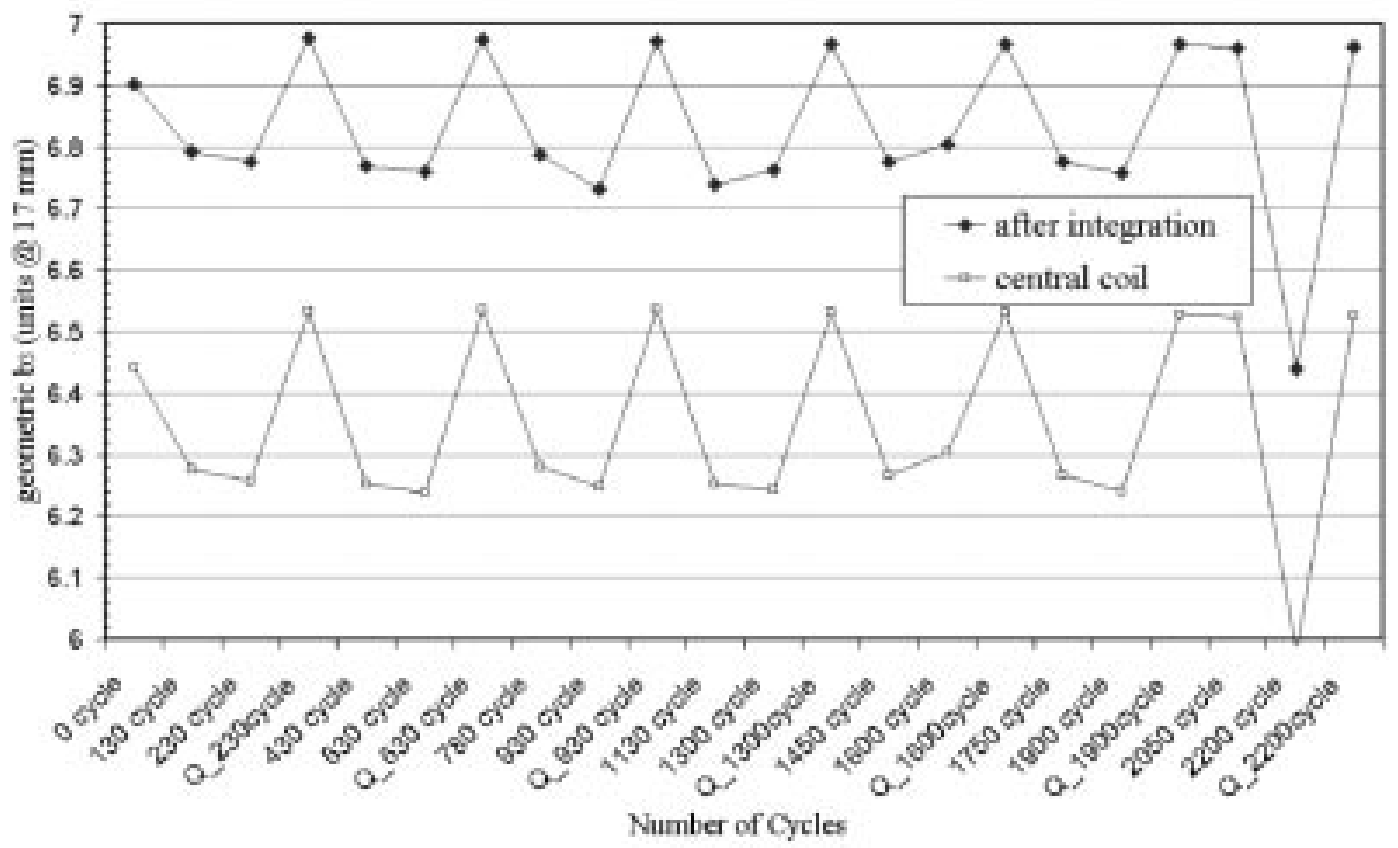

Fig. 8. Lifetime variations of the geometric sextupole (example).

TABLE IV

\begin{tabular}{lcccc}
\hline MAGNET & $\Delta \mathrm{b} 2$ & $\Delta \mathrm{b} 3$ & $\Delta \mathrm{b} 5$ & $\Delta \mathrm{b} 7$ \\
\hline MBSMT4v1a1 & 0.22 & 0.27 & 0.13 & 0.05 \\
MBSMT4v1a2 & 0.21 & 0.61 & 0.11 & 0.17 \\
MBSMT4v2a1 & 0.28 & 0.58 & 0.20 & 0.09 \\
MBSMT4v2a2 & 0.15 & 0.36 & 0.12 & 0.04 \\
MBSMT5v1a1 & 0.16 & 0.63 & 0.09 & 0.03 \\
MBSMT5v1a2 & 0.12 & 0.22 & 0.07 & 0.04 \\
MBSMT5v2a1 & 0.25 & 0.79 & 0.05 & 0.04 \\
MBSMT5v2a2 & 0.19 & 0.63 & 0.07 & 0.04 \\
MBSMT9v1a1 & 0.17 & 0.21 & 0.07 & 0.02 \\
MBSMT9v1a2 & 0.69 & 0.72 & 0.01 & 0.13 \\
MBSMT10v1a1 & 0.14 & 0.22 & 0.09 & 0.09 \\
MBSMT10v1a2 & 0.11 & 0.13 & 0.10 & 0.01 \\
MBSMT10v3a1 & 0.06 & 0.17 & 0.04 & 0.01 \\
MBSMT10v3a2 & 0.16 & 0.12 & 0.03 & 0.01 \\
MBSMT11v1a2 & 0.08 & 0.22 & 0.05 & 0.03 \\
\hline
\end{tabular}

11750 A (lifetime cycles). After some hundreds of current cycles were accumulated, a magnetic measurement was performed, then the magnet was quenched, and the field was measured again. As a result of this procedure the geometric harmonics were systematically found to drift monotonically as a function of the current cycles, and to recover after quench, as shown in Fig. 8. The direction of the drifts was not systematic as in the training experiment. Some tests were dedicated to determine the effect of parameters such as the maximum current in the current cycles and the quench energy on the shifts. When the maximum current was lowered to $8350 \mathrm{~A}$, corresponding to half of the Lorenz force, the drift of b3 was reduced by about $20 \%$. On the contrary, the quench energy does not have a systematic influence on the observed amount of recovery.

\section{DISCUSSION}

\section{A. Field Reconstruction}

The field reconstruction presented in Section III-B relies on a simple model of the coil deformation, only taking into account the azimuthal stretches of the inner and of the outer layers to form the jacobian. Including the correction of the estimated coil size given by the collar expansion is an important ingredient of the model, improving significantly the final correlations. However, the sensitivity to other deformation modes can jeopardize the results for some of the multipoles. In particular b5 is highly sensitive to the radial modes, as reported in [9]. As one can see in Fig. 5, the points appear to be correlated, though with a slope different from 1 . This suggests that the jacobian is not complete, that is, not all the relevant deformation modes are taken into account.

\section{B. Stability}

During training, the geometric normal sextupole invariably follows a rising curve reaching saturation after the first few quenches. The opposite trend is displayed by the geometric normal decapole (see Figs. 6 and 7). The effect, though small, is well above the measurement sensitivity $\left(10^{-2}\right.$ units). It qualitatively corresponds to a reduction in azimuthal coil length. Indeed the electromagnetic forces tend to compress the coil toward the mid-plane. According to the slopes presented in Table III, and considering only one deformation mode, a few tents micrometers compression is enough to explain the measured shift of geometric b3 and b5. After training the values are stabilized. However, a nonmonotonic behavior was observed in those magnets where the initial rise was larger. The effect of current cycles after training seems to be of different origin. The harmonic shift dependence on the maximum current in the cycles is closer to a linear one, than to the quadratic law 
expected from Lorenz forces. Moreover, the energy dissipated in the magnet during quenches is not an important parameter. The transition to the normal state, with the consequent erasing of all persistent currents, is the most probable explanation for the multipole recovery after quenches in lifetime tests. Actually, as a result of the particular test procedure, these measurements were all taken in different pre-cycling conditions, so it is likely that the shifts are due to electromagnetic effects, rather than to a real deformation of the coils.

\section{CONCLUSION}

The magnetic field quality of eight twin aperture superconducting short dipole models was measured in warm and cold conditions. A good warm cold correlation has been found and the geometric field errors have been recalculated from shimming and pre-stress data as in previous works. The field reconstructions are quite satisfactory for $\mathrm{b} 3$ and $\mathrm{b} 7$, while they indicate that a different deformation mode, not taken into account in this simple model, contributes to the measured b5.

During magnet training, the mechanical adjustments occurring in superconducting coils produce variations of the geometric field quality. The effect of current cycles after training, instead, should be regarded as a measurement artifact peculiar to the short models, which would not be observed in the long magnets.

\section{ACKNOWLEDGMENT}

The authors would like to thank all the former and present members of the B4 and Bldg. 927 teams.

\section{REFERENCES}

[1] S. Sanfilippo, A. Siemko, D. Tommasini, and W. Venturini, "Training quench performance and quench location of the short superconducting dipole models for the LHC,", submitted for publication.

[2] Z. Ang, I. Bejar, L. Bottura, D. Richter, M. Scheehan, L. Walckiers, and R. Wolf, "Measurement of AC loss and magnetic field during ramps in the LHC model dipoles," IEEE Trans. Appl. Superconduct., vol. 9, pp. 742-745, 1999.

[3] K. H. Mess, P. Schmüser, and S. Wolff, Superconducting accelerator magnets. Singapore: World Scientific, 1996, p. 45.

[4] L. Bottura, "Field dynamics in superconducting magnets for particle accelerators," in Proc. CERN Accelerator School (CAS), Geneva, 1998, p. 79.

[5] M. Alexa, S. Amet, Z. Ang, L. Bottura, M. Buzio, P. Ferracin, O. Pagano, V. Remondino, S. Russenschuck, S. Sanfilppo, W. Scandale, and E. Todesco, "Measurement and analysis of the field quality of the LHC superconducting dipoles,", submitted for publication.

[6] Z Ang, L. Bottura, S. Russenschuck, A. Siemko, D. Tommasini, and L. Walckiers, "Coil size and geometric field quality in short model dipoles for LHC," IEEE Trans. Appl. Superconduct., vol. 10, pp. 65-68, 2000.

[7] P. Ferracin, W Scandale, E. Todesco, and D. Tommasini, "Modeling of the coil pre-stress loss during cool down in the main dipoles of the Large Hadron Collider,", submitted for publication.

[8] L. Bottura, CERN, CH-1211 Geneva 23, Switzerland, 2000.

[9] P. Ferracin, O. Pagano, V. Remondino, W. Scandale, E. Todesco, and D. Tommasini, "Impact of coil deformations on field quality in the Large Hadron Collider main dipole,", submitted for publication. 